Herz 2019 · 44:287-288

https://doi.org/10.1007/s00059-019-4810-y

Online publiziert: 30. April 2019

(c) Springer Medizin Verlag GmbH, ein Teil von Springer Nature 2019

\section{R. Erbel' $\cdot$ H.-C. Diener ${ }^{2}$}

'Institut für Medizinische Informatik, Biometrie und Epidemiologie (IMIBE), Universitätsklinikum Essen (AöR), Essen, Deutschland

${ }^{2}$ Klinik für Neurologie, Universitätsklinikum Essen (AöR), Essen, Deutschland

\title{
Herz und Hirn - zwei Begriffe, ein zentrales Problem
}

\section{Der Schlaganfall}

Die Deutsche Gesellschaft für Innere Medizin, zunächst 1882 organisiert über den „Congress für Innere Medizin“ und 1920 umgewandelt in „Deutsche Gesellschaft für Innere Medizin“, war lange Zeit die Muttergesellschaft, aus der 1907 die „Gesellschaft Deutscher Nervenärzte“ hervorging, die seit 1950 den Namen „Deutsche Gesellschaft für Neurologie" trägt. Erst 1927 wurde die „Deutsche Gesellschaft für Kreislaufforschung "gegründet, die 1979 in „Deutsche Gesellschaft für Herz- und Kreislaufforschung " und 1994 in „Deutsche Gesellschaft für Kardiologie - Herz- und Kreislaufforschung" umbenannt wurde. Noch vor 50 Jahren erschien das Lehrbuch „Grundbegriffe der Inneren Medizin und Neurologie" von A. Sturm, der als Professor für Innere Medizin an der Universität Düsseldorf lehrte und gleichzeitig als Direktor der Städtischen Krankenanstalten Wuppertal-Barmen wirkte [1]. In der Folgezeit führte aber der Weg der beiden genannten Gesellschaften in unterschiedliche Richtungen, und wenige gemeinsame Forschungsinhalte wurden bearbeitet. Dies änderte sich mit der verbesserten Funktionsdiagnostik und der modernen interventionellen Therapie des Herzens. Diese Ausgabe von Herz (4/2019) widmet sich dem Thema "Herz und Hirn“, dem sich beide Herausgeber bereits mit ihrer früheren klinischen Arbeit am Universitätsklinikum Essen und auch jetzt im Institut für Medizinische Informatik, Biometrie und Epidemiologie der Medizinischen Fakultät in Essen noch immer wissenschaftlich verbunden fühlen. Eine gemeinsame Autorenschaft aus der Kar- diologie und Neurologie sollte die neue Brücke aufzeigen, die zwischen diesen beiden Disziplinen zum Wohl der Patienten gebaut wurde.

Die Diagnostik des Vorhofflimmerns (VHF) hat eine hohe Bedeutung für beide Fächer, und zwar in Bezug auf sowohl die Diagnostik als auch die Therapie. Aus Leipzig und Bielefeld kommen die Autoren Stegmann, Wachter und Schäbitz, die das zentrale Thema „ESUS (,embolic stroke of undetermined source $\left.{ }^{c}\right)$ - Wie viel Monitoring ist nötig?" bearbeitet haben. Sie stellen die Datenlage und die sich daraus ableitbaren Empfehlungen zur Patientenselektion und zur abgestuften Rhythmusdiagnostik sowie das diagnostische Stufenschema zur Dauer des EKG-Monitorings bei Patienten mit Schlaganfall der Arbeitsgemeinschaft Herz und Hirn der Deutschen Gesellschaft für Kardiologie - Herzund Kreislaufforschung e. V. (DGK) und der Deutschen Schlaganfall-Gesellschaft e. V. (DSG) vor [2].

Neben der vielfältigen EKG-Diagnostik spielt die Bildgebung - die Echokardiographie, die Computertomographie (CT) und die Magnetresonanztomographie (MRT) - eine entscheidende Rolle, die vom Hamburger und Würzburger Team um Camen, Häusler und Schnabel vorgestellt wird. Die Einführung der transösophagealen Echokardiographie (TEE) erlaubte der klinischen Medizin erstaunliche neue Einblicke ins Herz und seine Strukturen sowie den Nachweis von Vegetationen bei einer Endokarditis an noch intakten Klappen [3]. Schlaganfälle werden in $15-29 \%$ der Fälle mit Endo- karditis beobachtet [4]. Bei etwa 3\% der Schlaganfälle wird bei jungen Menschen eine Endokarditis als Ursache aufgedeckt [5]. War schon die Aufdeckung von Aneurysmen des Vorhofseptums [6] eine Überraschung, allerdings in der Pathologie durchaus bekannt [7], war der Nachweis der paradoxen Embolie mit Darstellung eines Thrombus, gefangen in einem offenen Foramen ovale (PFO; Vorhofseptum), eine Sensation [8] und eröffnete die Diskussion um den Sinn eines PFO-Verschlusses $[9,10]$. Die Diskussion führte zu wichtigen Entscheidungen und klaren Empfehlungen [11], die von Diener und Knebel aus Essen und Berlin diskutiert werden. Die TEE-Untersuchungen bei Patienten mit VHF führte auch zu der Erkenntnis, dass im linken Vorhofohr (,left atrial appendage", LAA), also in einem bis dahin eher weniger beachteten Herzabschnitt, eine wichtige kardiale Emboliequelle lag. In knapp $14 \%$ der Fälle wurden Thromben im Herzen nachgewiesen, die zu fast $90 \%$ im LAA nachweisbar waren [12]. Der Berner Kardiologe B. Meier, ein früher und starker Verfechter des PFO-Verschlusses [13], war auch an der Entwicklung eines Verschlusssystems für das LAA beteiligt [14]. Die Kollegen aus Würzburg und Berlin, Häussler und Landmesser, stellen den derzeitigen Stand der Forschung vor und geben einen Ausblick auf die derzeitige Studienlage zum LAA-Verschluss.

Die Fortschritte der CT- und MRTDiagnostik erfüllten wichtige Hoffnungen der Neurologie hinsichtlich der sicheren Diagnose und Abgrenzung von 
ischämischen und nichtischämischen Insulten - eine Voraussetzung für weitere Therapieentscheidungen. Neben VHF spielt eine Reihe anderer Diagnosen, wie z. B. Herztumoren, eine wichtige Rolle, für deren Aufdeckung eine Bildgebung mit großer räumlicher Auflösung wie die CT und die MRT wichtig ist. Mit den modifizierten TOAST(Trial of Org 10172 in Acute Stroke Treatment)-Kriterien liegt eine anerkannte Klassifizierung vor, die in der Bildgebung umgesetzt werden sollte $[15,16]$.

Ein besonderes therapeutisches Problem in der Therapie des VHF greifen die Kollegen aus Erlangen und Frankfurt, Sembill, Kuramatsu, Hohnloser und Huttner, auf. Wenn auch die Rolle der Antikoagulation in der Therapie des VHF unumstritten ist, bleibt die Beherrschung von Blutungskomplikationen ein großes interdisziplinäres Problem. Die Einführung spezifischer Antikörper bedeutete einen entscheidenden Durchbruch für die Sicherheit der Patienten, da eine Blutungsneigung in Sekunden gestoppt werden kann. Die Nutzung von standardisierten Therapieschemata ist bei den seltenen, aber dramatischen Komplikationen der VHF-Therapie extrem wichtig. Die Autoren stellen dazu ein sehr gutes Ablaufschemata zur Verfügung.

Die Herausgeber hoffen sehr, dass Ihnen diese Ausgabe von Herz, die dem Thema „Herz und Hirn“ gewidmet ist und gemeinsam von Kardiologen und Neurologen verfasst wurde, gefällt, und wünschen eine interessante Lektüre mit vielen Anregungen zur Diskussion.

\section{Korrespondenzadresse}

\section{Prof. Dr. med. R. Erbel}

Institut für Medizinische Informatik, Biometrie und Epidemiologie (IMIBE), Universitätsklinikum Essen (AöR) Hufelandstraße 55, 45147 Essen, Deutschland erbel@uk-essen.de

Förderung. Forschungsprojekte der Universitätsklinik für Neurologie in Essen wurden unterstützt von AstraZeneca, GlaxoSmithKline, Boehringer Ingelheim, Novartis, Janssen-Cilag und Sanofi-Aventis. Die Universitätsklinik für Neurologie hat Forschungsmittel von den folgenden Institutionen erhalten: Deutsche Forschungsgemeinschaft (DFG), Bundesministerium für Bildung und Forschung (BMBF), Europäische Union (EU), National Institutes of Health (NIH), EAST (Early Treatment of Atrial Fibrillation for
Stroke Prevention Trial)-AFNET (Atrial Fibrillation Network), Bertelsmann Stiftung und Heinz Nixdorf Stiftung.

Interessenkonflikt. R. Erbel gibt an, dass kein Interessenkonflikt besteht. H.-C. Diener hat Honorare für Teilnahme an klinischen Studien, Mitarbeit in Advisory Boards und Vorträge erhalten von Abbott, Allergan, AstraZeneca, Bayer Vital, Bristol-Myers Squibb, Boehringer Ingelheim, BrainsGate, CoAxia, Corimmun, Covidien, Daiichi Sankyo, D-Pharm, Fresenius, GlaxoSmithKline, Janssen-Cilag, Lilly, Lundbeck, Medtronic MSD, MindFrame, Neurobiological Technologies, Novartis, Novo Nordisk, Paion, Parke-Davis, Pfizer, Portola, Sanofi-Aventis, Schering, Servier, Solvay, St. Jude Medical, Syngis, Tacrelis, Thrombogenics und Wyeth. H.-C. Diener besitzt keine Aktien oder Anteile von Pharma- oder Medizintechnikfirmen und war beteiligt an der Erstellung von Leitlinien der Deutschen Gesellschaft für Neurologie (DGN), der Deutschen Schlaganfall-Gesellschaft (DSG), der European Society of Cardiology (ESC) und der European Heart Rhythm Association (EHRA).

\section{Literatur}

1. Sturm A (1968) Grundbegriffe der Inneren Medizin und Neurologie, 2. Aufl. Gustav Fischer, Stuttgart

2. Häusler K, Gröschel K, Köhrmann M et al (2018) Positionspapier zur Detektion von Vorhofflimmern nach ischämischem Schlaganfall: Arbeitsgemeinschaft Herz und Hirn der Deutschen Gesellschaft für Kardiologie - Herz- und Kreislaufforschung e.V. (DGK) und der Deutschen SchlaganfallGesellschafte. V. (DSG). Aktuelle Neurol 45:93-106

3. Erbel R, RohmannS, DrexlerMetal (1988) Improved diagnostic value of echocardiography in patients with infective endocarditis by transoesophageal approach. A prospective study. Eur Heart J 9:43-53

4. García-Cabrera E, Fernández-Hidalgo N, Almirante $B$ et al (2018) Neurological complications of infective endocarditis: risk factors, outcome, and impact of cardiac surgery: a multicenter observational study. Circulation 127:2272-2284

5. Yesilot Barlas N, Putaala J, Waje-Andreassen U et al (2013) Etiology of first-ever ischaemic stroke in European young adults: the 15 cities young stroke study. Eur J Neurol 20:1431-1439

6. Schreiner G, Erbel R, Mohr-Kahaly S, Krämer G, Henkel B, Meyer J (1985) Nachweis von Aneurysmen des Vorhofseptums mit Hilfe der transösophagealen Echokardiographie. Z Kardiol 74:440-444

7. Silver MD, Dorsey JS (1978) Aneurysm of the septum primum in the adults. Arch Path Lab Med 102:62-65

8. Nellessen U, Daniel WG, Matheis G, Oelert H, Depping K, Lichtlen PR (1985) Impending paradoxical embolism from atrial thrombus: correct diagnosis by transesophageal echocardiography and prevention by surgery. J Am Coll Cardiol 5:1002-1004

9. Hausmann D, Mügge A, Becht I, Daniel WG (1992 Diagnosis of patent foramen ovale by transesophageal echocardiography and association with cerebral and peripheral embolic events. Am J Cardiol 70:668-672

10. Van Camp G, Schulze D, Cosyns B, Vandenbossche JL (1993) Relation between patent foramen ovale and unexplained stroke. Am J Cardiol 71:596-598

11. Diener HC, Grau AJ, Baldus S et al (2018) Kryptogener Schlaganfall und offenes Formaen ovale: S2e-Leitlinie [Cryptogenic stroke and patent foramen ovale : S2eguidelines. Nervenarzt 89:1143-1153

12. Klein AL, Grimm RA, Murray RD et al (2001) Use of transesophageal echocardiography to guide cardioversion in patients with atrial fibrillation. NEngl J Med 344:1411-1420

13. Meier B (2009) Catheter-based closure of the patent foramen ovale. Circulation 120:1837-1841

14. Meier B, Palacios I, Windecker S et al (2003) Transcatheter left atrial appendage occlusion with Amplatzer devices to obviate anticoagulation in patients with atrial fibrillation. Catheter Cardiovasc Interv 60:417-422

15. Goldstein LB, Jones MR, Matchar DB et al (2001) Improving the reliability of stroke subgroup classification using the Trial of ORG 10172 in Acute Stroke Treatment (TOAST) criteria. Stroke 32:1091-1098

16. Saric M, Armour AC, Arnaout MS et al (2016) Guidelines for the Use of Echocardiography in the Evaluation of a Cardiac Source of Embolism. J Am Soc Echocardiogr 29:1-42 